LA WAENCE LIVEAMCAE NATIONAL LABOAATOAY

\title{
Calibration of a Flat Field Soft $X$ ray Grating Spectrometer for Laser Produced Plasmas
}

J. Park, G. V. Brown, M. B. Schneider, H. A. Baldis, P. Beiersdorfer, K. V. Cone, R. L. Kelley, C. A. Kilbourne, E. W. Magee, M. J. May, and F. S. Porter

May 13, 2010

High Temperature Plasma Diagnostics 2010 Wildwood, NJ, United States May 16, 2010 through May 20, 2010 
This document was prepared as an account of work sponsored by an agency of the United States government. Neither the United States government nor Lawrence Livermore National Security, LLC, nor any of their employees makes any warranty, expressed or implied, or assumes any legal liability or responsibility for the accuracy, completeness, or usefulness of any information, apparatus, product, or process disclosed, or represents that its use would not infringe privately owned rights. Reference herein to any specific commercial product, process, or service by trade name, trademark, manufacturer, or otherwise does not necessarily constitute or imply its endorsement, recommendation, or favoring by the United States government or Lawrence Livermore National Security, LLC. The views and opinions of authors expressed herein do not necessarily state or reflect those of the United States government or Lawrence Livermore National Security, LLC, and shall not be used for advertising or product endorsement purposes. 


\title{
Calibration of a Flat Field Soft X ray Grating Spectrometer for Laser Produced Plasmas
}

\author{
J. Park ${ }^{1,2, b)}$ G. V. Brown, ${ }^{1}$ M. B. Schneider, ${ }^{1}$ H. A. Baldis, ${ }^{2}$ P. Beiersdorfer, ${ }^{1}$ K. V. \\ Cone,${ }^{1,2}$ R. L. Kelley, ${ }^{3}$ C. A. Kilbourne,${ }^{3}$ E. W. Magee,${ }^{1}$ M. J. May, ${ }^{1}$ and F. S. \\ Porter $^{3}$
}

${ }^{1}$ Lawrence Livermore National Laboratory, P.O. Box 808, Livermore, CA 94551-0808

${ }^{2}$ University of California, Davis, One Shields Avenue, Davis, CA 95616

${ }^{3}$ NASA Goddard Space Flight Center, Greenbelt, MD 20770

\begin{abstract}
We have calibrated the $\mathrm{x}$ ray response of a variable line spaced grating spectrometer, known as the VSG, at the Fusion and Astrophysics Data and Diagnostic Calibration Facility at the Lawrence Livermore National Laboratory (LLNL). The VSG has been developed to diagnose laser produced plasmas, such as those created at the Jupiter Laser Facility and the National Ignition Facility at LLNL, and at both the Omega and Omega EP lasers at University of Rochester's Laboratory for Laser Energetics. The bandwidth of the VSG spans the range from $\sim 6$ to $60 \AA$. The calibration results present here include the VSG's dispersion and quantum efficiency. The dispersion is determined by measuring the $\mathrm{x}$ rays emitted from hydrogen-like and helium-like ions of carbon, nitrogen, oxygen, neon, and aluminum. The quantum efficiency is calibrated to an accuracy of $30 \%$ or better by normalizing the $\mathrm{x}$ ray intensities recorded by the VSG to those simultaneously recorded by an $\mathrm{x}$ ray microcalorimeter spectrometer.
\end{abstract}

\section{INTRODUCTION}

High resolution grating and crystal spectrometers have been employed to diagnose high temperature plasmas for several decades. Recently, they have been used at the Jupiter Laser Facility at the Lawrence Livermore National Laboratory (LLNL) and at both Omega and Omega EP at the University of Rochester's Laboratory for Laser Energetics (LLE) to measure the electron temperature and density. ${ }^{1}$ The utility of these instruments is determined by the knowledge of the response of the instrument to incident $x$ rays, i.e., knowledge of the overall quantum efficiency and the dispersion. We have used the Fusion and Astrophysics (FAST) Data and Diagnostic Calibration Facility $^{2}$ at LLNL to determine the absolute quantum efficiency and the dispersion for a variable line space grating spectrometer, known as the VSG. Here we describe the basic properties of the spectrometer, the calibration methods, and our results.

\section{CALIBRATION ARRANGEMENT}

The FAST calibration facility is centered around the EBIT-I electron beam ion trap, ${ }^{3,4}$ designed and built at the LLNL, and the EBIT Calorimeter Spectrometer ${ }^{5}$ (ECS) designed and built at the NASA/Goddard Space Flight Center. EBIT-I uses an electron beam and an electrostatic trap to confine and create highly charged ions from any element. Ions of nearly every element have been produced. Once trapped, the ions are excited by the electron beam, and $\mathrm{x}$ rays are produced via radiative stabilization. The diameter of the electron beam is approximately $60 \mu \mathrm{m}$, and thus the beam itself acts as a slit for grating and crystal spectrometers. Access to the trap is provided by six vacuum ports looking directly at the trap region. One of these ports is home to the EBIT diagnostic instrument manipulator (DIM) tube, as illustrated in Fig. 1. The EBIT DIM tube was designed to house nearly any spectrometer that can be used in a typical DIM tube at the National Ignition Facility ${ }^{6}$ (NIF) or ten-inch manipulator (TIM) tube at Omega. Similar to its operation at LLE's Omega,

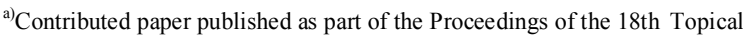
Conference on High-Temperature Plasma Diagnostics, Wildwood, New Jersey, May, 2010

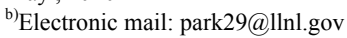

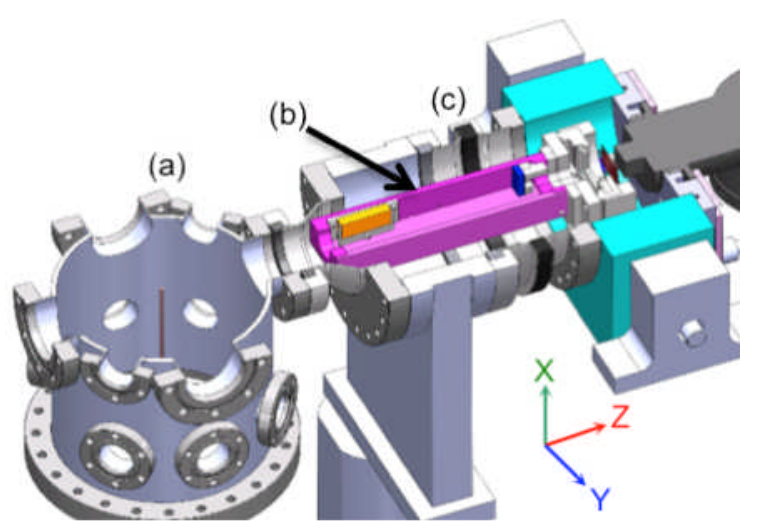

FIG. 1. The VSG (b) is mounted inside of the EBIT DIM tube (c) connected to the EBIT vacuum chamber (a). The VSG in this diagram is translated to its calibration position.

the VSG is mounted in the EBIT DIM tube and then translated under vacuum towards the beam until it reaches its operating distance. Fig. 1 shows a schematic of the VSG inside the EBIT DIM at its operating distance.

The VSG was designed and built at the LLNL to measure the L-shell spectra of near neon-like ions of titanium to copper produced in laser plasmas. ${ }^{7,8}$ It has been regularly used at the COMET $^{9}$ laser at LLNL's Jupiter Laser Facility, and at the Omega Laser facility ${ }^{10}$ at the University of Rochester. The VSG consists of a diffraction grating, a spectrometer housing, and a removable nose cone that can house slits for spatially resolved measurements. The grating has variably spaced grooves designed to focus diffracted light onto a flat field. ${ }^{11}$ This makes it possible to use a flat detector without suffering an appreciable loss of resolution across the covered spectral range. The grating has a nominal line spacing of 2400 lines $/ \mathrm{mm}$ and is optimized for an $1.3^{\circ}$ angle of incidence relative to the grating plane. In the VSG housing, the grating is tilted at an angle of $1.8^{\circ}$ to the horizontal and toward the detector in order to keep the line from the source 
to the center of the image plane coincident with the central axis of the VSG and still maintain a $1.3^{\circ}$ grazing angle of incidence on the grating. This geometry allows us to easily operate the VSG in any vacuum port with any rotation at Omega, Omega EP, or NIF. The $38 \mathrm{~mm}$ wide image plane provides spectral coverage up to $62 \AA$. The VSG has an objective distance of $236 \mathrm{~mm}$ (source to grating center) and the distance from the source to the center of the detector is $472 \mathrm{~mm}$. For these measurements, the VSG spectrometer is mounted so that the grooves of the grating are aligned parallel to the vertical electron beam. The VSG is designed for use with film, image plates, or $\mathrm{x}$ ray cameras. A liquid nitrogen cooled back-illuminated $\mathrm{x}$ ray charge coupled device (CCD) is used for this calibration. The CCD has $1300 \times$ 1340 pixels that are of $20 \times 20 \mu \mathrm{m}^{2}$ size. $^{12}$ To filter out unwanted stray optical, vacuum ultraviolet, and extreme ultraviolet light, a $2000 \AA$ thick lexan filter coated with $1500 \AA$ of aluminum is placed in front of the CCD. This filter has been calibrated earlier. ${ }^{13}$ No nose cone is used here because no spatial resolution is necessary and the photon flux from EBIT would be greatly reduced. The solid angle of the instrument in this arrangement is $2.66 \times 10^{-4} \mathrm{sr}$.

Just like in the case of the DIM tube at NIF and the TIM tube at Omega and Omega EP, the distance from the grating to the $\mathrm{x}$ ray source (the center of EBIT-I where the electron beam is located) is set using a precision machined pointer. The distance from the tip of the pointer to the center of the grating is equal to the object distance of $236 \mathrm{~mm}$. To align the VSG, the pointer is mounted on the front of the VSG. The VSG with the pointer is installed in the EBIT DIM tube, and then the VSG is translated until the tip of the pointer is at the center of EBIT-I. The position is verified by sighting the pointer tip inside EBIT-I through a vacuum port outfitted with an optical window and a telescope on top of the device. This entire process is completed under vacuum $\left(\sim 10^{-9}\right.$ torr $)$ and while EBIT-I's trap electrodes are at cryogenic temperatures.

The quantum efficiency (QE) of the VSG is determined by normalizing to the ECS. The ECS consists of an array of 32 individual pixels each mounted with a $\mathrm{HgTe}$ absorber for stopping $\mathrm{x}$ rays. Eighteen of the channels are optimized for the detection of photon with energies between 0.1 and $15 \mathrm{keV}$, and
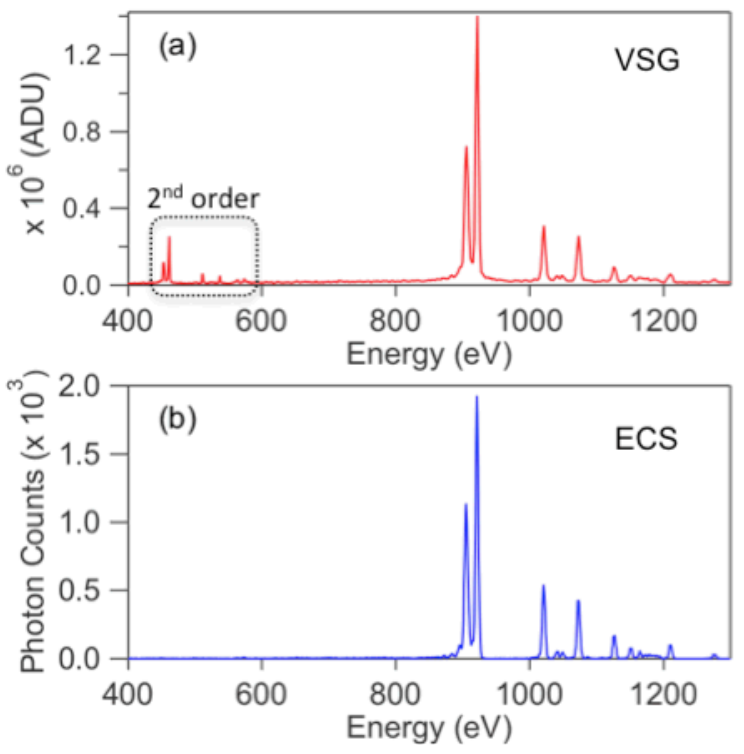

FIG. 2. X ray line emission from helium-like Ne IX and hydrogenic Ne X. Spectra recorded by the (a) VSG and the (b) ECS are shown. The $2^{\text {nd }}$ order diffraction in the VSG is also shown. thirteen are optimized for photons between 15 and $100 \mathrm{keV}$. The low energy channels used here have absorbers with an area of $0.624 \times 0.624 \mu \mathrm{m}^{2}$ and a thickness of $8 \mu \mathrm{m}$. The energy resolution of the lower energy channels is $\sim 4.5 \mathrm{eV}$ at $6 \mathrm{keV}$. In order to detect single photons with high resolution, the ECS detector array operates at a temperature of 50 milliKelvin. The ECS detector is situated $95.17 \mathrm{~cm}$ from the center of the trap and each pixel has a solid angle of $4.3 \times 10^{-7} \mathrm{sr}$. To reduce the stray heat load and unwanted noise on the detector, it is shielded from thermal radiation by four individual aluminized polyimide filters situated at different temperature stages between the detector plane and the dewar's vacuum jacket. The total thickness of theses filters is $1470 \AA$ of aluminum and $2386 \AA$ of polyimide. Furthermore, there is a fifth aluminized polyimide window $(10,300 \AA$ of polyimide and $200 \AA$ of aluminum) housed in a valve between the ECS and the EBIT-I gate valve. This can be inserted into the line of sight between EBIT-I's trap region and the ECS detector to eliminate unwanted low energy photon flux. The quantum efficiency of the absorbers has been determined as part of the XRS flight instrument calibration program, ${ }^{14}$ and the $\mathrm{x}$ ray transmittance as a function of energy for the blocking filters is given by the Center for $\mathrm{X}$ ray Optics (http://henke.lbl.gov/optical_constants/).

To determine the VSG's dispersion and absolute quantum efficiency as a function of energy, we used the line emission from the hydrogen and helium like ions of carbon, nitrogen, oxygen, neon, and aluminum. All these ions are easily created and trapped in EBIT-I and are routinely used as reference standards, ${ }^{15}$ as the wavelengths of all the lines used here are well known. Typical spectra of helium-like Ne IX and hydrogenic Ne X measured simultaneously by the ECS and the VSG are shown in Fig. 2.

The dispersion curve for the VSG was found by measuring the centers of the $\mathrm{x}$ ray lines from each of the highly charged ions mentioned above, fitting a Gaussian curve to each line to determine its centroid in CCD pixel space, converting the pixel number to distance along the image plane, and plotting the wavelength of the lines versus their centroids in $\mathrm{mm}$, and fitting the curve. The result is shown in figure 3 . The equation used to fit the curve is the standard grating equation given by:

$$
m \lambda=d(\sin \alpha-\sin \beta),
$$

where $m$ is the diffraction order, $d$ the line spacing in the center of the grating, $\alpha$ the normal incident angle $88.7^{\circ}$, and $\beta$ the dispersion angle given by $\left[\arctan \left(236 /\left(\mathrm{y}-\mathrm{y}_{0}\right)\right)-1.8^{\circ}\right]$ where $\mathrm{y}$ is the spectral line position in millimeters and $\mathrm{y}_{0}$ is the offset.

The absolute efficiency of the VSG spectrometer is determined by measuring the spectra emitted from the EBIT-I simultaneously with both the VSG and the ECS, and then normalizing the number of photons detected in the VSG to the number of photons detected by the ECS. Once normalized, the reflectivity of the VSG grating can be determined. The equations for the number of photons detected by the VSG and the ECS, respectively, are:

$$
\begin{aligned}
& N_{V S G}=\varepsilon \times T C_{V S G} \times V_{V S G} \times t \times \Delta \Omega_{V S G} \times Q E_{C C D} \times R_{\text {gratine }} \\
& N_{E C S}=\varepsilon \times T C_{E C S} \times V_{E C S} \times t \times \Delta \Omega_{E C S} \times Q E_{E C S}
\end{aligned}
$$

where $\varepsilon$ is the emissivity defined here as photons per second per $\mathrm{cm}^{3}, N$ the number of detected photons, $T C$ the transmission efficiency for the filters, $V$ the volume of the $\mathrm{x}$ ray source seen by spectrometers, $t$ the exposure time, $\Delta \Omega$ the subtended solid angle, 


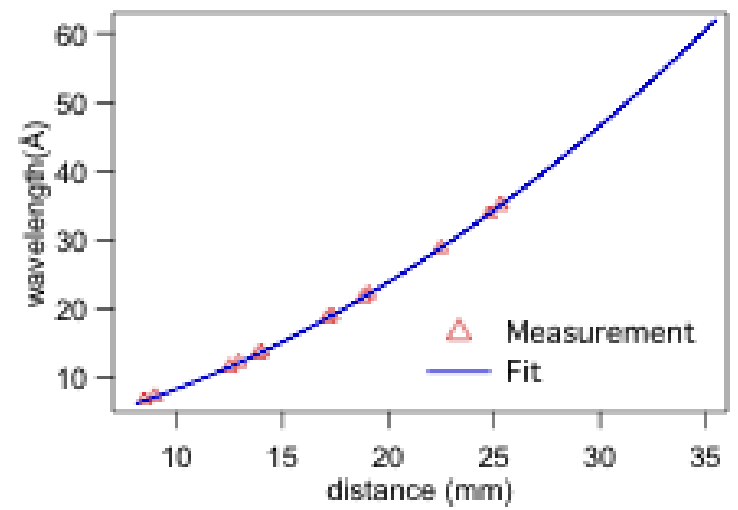

FIG. 3. The measured $(\Delta)$ and the fitted (line) dispersions of the VSG are compared.

$Q E$ the quantum efficiency of the detectors, and $R_{\text {grating }}$ the reflectivity of the grating.

We note that the CCD does not the record photon number directly. It records ADU counts which can be converted to photon number using the formula, $N=A D U \times G \times W / E$, where the gain, $G$, is given by the manufacturer to be 1.05 electrons/ADU, $W$ is the work function of $\mathrm{Si}(3.65 \mathrm{eV} /$ electron), and $E$ is the energy of the incident photon in $\mathrm{eV}$.

By normalizing the number of photons detected by the VSG to the number of photons detected by the ECS and solving for the reflectivity of the grating we have:

$$
R_{\text {grating }}=\frac{N_{V S G}}{N_{E C S}}\left(\frac{\Delta \Omega_{E C S}}{\Delta \Omega_{V S G}}\right)\left(\frac{T C_{E C S}}{T C_{V S G}}\right)\left(\frac{Q E_{\text {absorber }}}{Q E_{C C D}}\right)\left(\frac{V_{E C S}}{V_{V S G}}\right) .
$$

\section{RESULT}

Figure 3 shows the measured dispersion compared with the theoretical curve described by the grating equation. Not surprisingly, the grating equation nicely represents the data.

Figure $4 \mathrm{a}$ shows the measured percent quantum efficiency of the full VSG instrument including the CCD camera, and Figure $4 \mathrm{~b}$ shows the percent reflectivity of the grating by itself. The
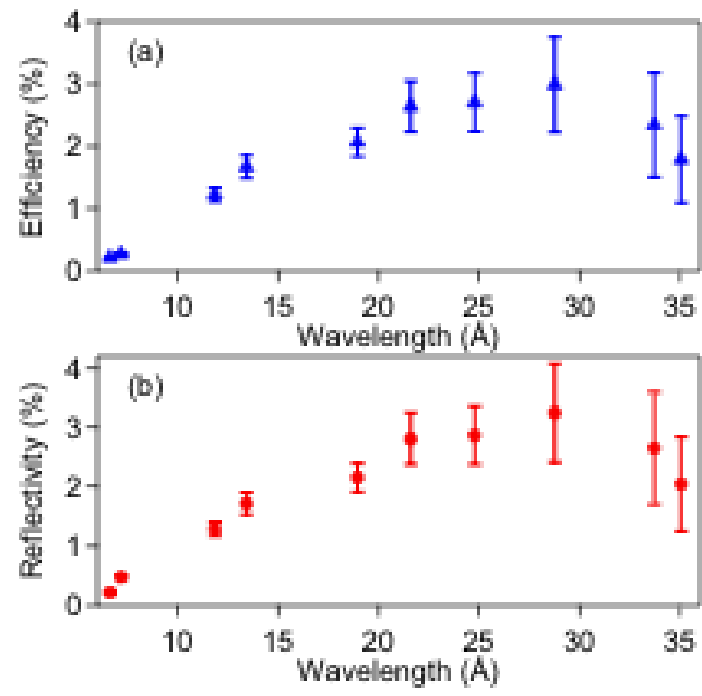

FIG. 4. The response of the VSG is shown: (a) the efficiency of the VSG including the CCD camera, and (b) the reflectivity of the grating only. quantum efficiency of the CCD camera is determined by assuming typical values of the dead layer, i.e., $10 \mathrm{~nm}$ of $\mathrm{SiO}_{2}$ and $20 \mathrm{~nm}$ of $\mathrm{Si}$, and $10 \mu \mathrm{m}$ of $\mathrm{Si}$ for an active layer and calculating the efficiency according to the method given by Li, Tsakiris, and Sigel. ${ }^{16}$

The error bars in Fig. 4 are mainly due to the uncertainty in the thicknesses of filters given by the manufacturer. Measurements of each of these filter thicknesses is underway and we expect the errors to be reduced once those measurements are complete. In addition we plan to directly measure the quantum efficiency of the CCD camera on the FAST facility and also perform detailed studies of the VSG line shape. We note that other spectrometers designed for use at NIF, Omega, or Omega EP that fit into the DIM or TIM envelopes can also be easily calibrated using this same method.

\section{ACKNOWLEDGEMENT}

This work was performed under the auspices of the U.S. Department of Energy by the Lawrence Livermore National Laboratory under Contract DE-AC52-07NA27344 and LDRD 08-ERD-024.

\section{REFERENCE}

${ }^{1}$ R. F. Heeter, S. G. Anderson, R. Booth, G. V. Brown, J. Emig, S. Fulkerson, T. McCaryille, D. Norman, M. B. Schneider, and B. K. F. Young, Rev. Sci. Instrum. 79, 10E303 (2008)

${ }^{2}$ G. V. Brown, P. Beiersdorfer, J. Dunn, E. W. Magee, J. Park, F. S. Porter, M. B. Schneider, E. Träbert, R. L. Kelley, and C. A. Kilbourne, Proceedings of the SPIE Astronomical Telescopes and Instrumentation 2010 Conference (unpublished)

${ }^{3}$ P. Beirsdorfer, Can. J. Phys., 86, 1 (2008)

${ }^{4}$ R. E. Marrs, Can. J. Phys., 86, 11 (2008)

${ }^{5}$ F. S. Porter, J. Gygax, R. L. Kelley, C. A. Kilbourne, J. M. King, P. Beiersdorfer, G. V. Brown, D. B. Thorn, S. M. Kahn, Rev. Sci. Instrum. 79, 307 (2008)

${ }^{6}$ E. I. Moses, R. N. Boyd, B. A. Remington, C. J. Keane, and R. Al-Ayat, Phys. Plasmas, 16, 041006 (2009)

${ }^{7}$ M. B. Schneider, D. E. Hinkel, S. J. Moon, S. B. Hansen, et al., High Energy Density Phys. 3, 256 (2007)

${ }^{8}$ K. V. Cone, J. Dunn, M. B. Schneider, H. A. Baldis, G. V. Brown, J. Emig, D. L. James, M. J. May, J. Park, and R. Shepherd, Rev. Sci. Instrum. (in these proceedings).

${ }^{9}$ Dunn, J. Nilsen, A. L. Osterheld, Y. Li, and V. Shlyaptsev, Opt. Lett. 24, 101 (1999)

${ }^{10}$ T. R. Boehly, D. L. Brown, R. S. Craxton, R. L. Keck, J. P. Knauer, J. H. Kelly, T. J. Kessler, S. A. Kumpan, S. J. Loucks, S. A. Letzring, F. J. Marshall, R. L. McCrory, S. F. B. Morse, W. Seka, J. M. Soures and C. P. Verdon, 133, 495 (1997)

${ }^{11}$ Toshiaki Kita, Tatsuo Harada, N. Nakano, and H. Kuroda, App. Opt., 22, 4 (1983)

${ }^{12}$ Roper Industries, Inc., Princeton Instruments, Model PI-SX 1300

${ }^{13}$ G. V. Brown, P. Beiersdorfer, J. Emig, M. Frankel, M. F. Gu, R. F. Heeter, E. Magee, D. B. Thorn, K. Widmann, R. L. Kelley, C. A. Kilbourne, and F. S. Porter, Rev. Sci. Instrum. 79, 10E309 (2008)

${ }^{14}$ R. L. Kelley, K. Mitsuda, C. A. Allen, P. Arsenovic, M. D. Audley, T. G. Bialas, K. R. Boyce, R. F. Boyle, S. R. Breon, G. V. Brown, J. Cottam, M. J. Dipirro, R. Fujimoto, T. Furusho, K. C. Gendreau, G. G. Gochar, O. Gonzales, M. Hirabayashi, S. S. Holt, H. Inoue, M. Ishida, Y. Ishisaki, C. S. Jones, R. Keski-Kuha, C. A. Kilbourne, D. McCammon, U. Morita, S. H. Moseley, B. Mott, K. Narasaki, Y. Ogawara, T. Ohashi, N. Ota, J. S. Panek, F. S. Porter, A. Serlemitsos, P. J. Shirron, G. A. Sneiderman, A. E. Szymkowiak, Y. Takei, J. L. Tveekrem, S. M Volz, M. Yamamoto, N. Y. Yamasaki, Publ. Astron. Soc. Japan 59, 77 (2007)

${ }^{15}$ P. Beiersdorfer, J. Phys. B 43, 074032 (2010)

${ }^{16}$ Yuelin Li, G. D. Tsakiris, and R. Sigel, Rev. Sci. Instrum., 66, 80 (1995) 